\title{
LA DIDÁCTICA DE LA GRAMÁTICA DESDE EL TRATAMIENTO INTEGRADO DE LENGUAS EN LA CAPV: ANÁLISIS DE MATERIALES DEL PROYECTO EKI
}

\author{
Recepción: 04/01/2020 | Revisión: 13/02/2020 | Aceptación: 11/04/2020
}

\section{Irene BALZA}

Universidad del País Vasco (UPV/EHU) irene.balza@ehu.eus

\author{
Ruth MILLA \\ Universidad del País Vasco (UPV/EHU) \\ ruth.milla@ehu.euss
}

\author{
M. Carmen ENCINAS REGUERO \\ Universidad del País Vasco (UPV/EHU) \\ mariadelcarmen.encinas@ehu.eus
}

\begin{abstract}
Resumen: Este trabajo constituye una revisión crítica de la manera en que se presentan los contenidos gramaticales en el proyecto didáctico EKI elaborado por la editorial IkasElkar para el último ciclo de Primaria. Dicho proyecto surge a raíz de la necesidad de editar materiales didácticos coherentes con el Decreto curricular 236/2015 para la Educación Básica de la comunidad autónoma del País Vasco (CAPV), en el marco del plan estratégico Heziberri 2020 para la mejora del sistema educativo vasco. Uno de los objetivos del proyecto es el multilingüismo y, para alcanzarlo, se recurre a un planteamiento integrado de lenguas que tiene como eje el euskara. El objetivo de este trabajo consiste en analizar cómo se inserta en dicho contexto la didáctica de la gramática. Tras el análisis de los materiales de Lengua Castellana, Euskara e Inglés, se concluye que los principios metodológicos son adecuados, aunque, en lo que a la enseñanza de la gramática se refiere, se echa en falta un mayor desarrollo de la reflexión metalingüística y un mayor aprovechamiento de la comparación entre lenguas.
\end{abstract}

Palabras clave: didáctica de la gramática; tratamiento integrado de lenguas; multilingüismo; plurilingüismo; reflexión metalingüística.

\section{THE DIDACTICS OF GRAMMAR WITHINTHE LANGUAGE INTEGRATEDTREATMENT INTHE BASQUE AUTONOMOUS COMMUNITY: ANALYSIS OF MATERIALS OFTHE EKI PROJECT}

\begin{abstract}
The present paper contains a critical review of the manner that grammar contents are presented in the EKI teaching project created by the IkasElkar publishing house for the last cycle of Primary Education. Such project arose from the necessity to create teaching materials that were in line with the curricular Decree 236/2015 for the Basic Education in the Basque Autonomous Community, framed by the strategic plan Heziberri 2020 for the improvement of the Basque educational system. One of the aims of the project is multilingualism and in order to achieve it, a language integrated approach was adopted, with Basque as the main axis. The present paper aims to analyse how grammar didactics is introduced in this context. After analysing the materials for Spanish, Basque and English, it seems that the methodological principles are appropriate, even though, as far as grammar teaching is concerned, it would be desirable to include further metalinguistic reflection and more explicit comparison between languages.
\end{abstract}

Keywords: didactics of grammar; language integrated treatment; multilingualism; plurilingualism; metalinguistic reflection.

\section{LA DIDÀCTICA DE LA GRAMÀTICA DES DEL TRACTAMENT INTEGRAT DE LLENGÜES A LA COMUNITAT AUTÒNOMA DEL PAÍS BASC: ANÀLISI DE MATERIALS DEL PROJECTE EKI}

Resum: Aquest treball constitueix una revisió crítica de la manera en què es presenten els continguts gramaticals al projecte didàctic EKI elaborat per l'editorial IkasElkar per a lúltim cicle de Primària. Aquest projecte sorgeix de la necessitat d'editar materials didàctics coherents amb el Decret curricular 236/2015 per a l'Educació Bàsica de la Comunitat Autònoma del País Basc, en el marc del pla estratègic Heziberri 2020 per a la millora del sistema educatiu basc. Un dels objectius del projecte és el multilingüisme i, per aconseguir-lo, es recorre a un plantejament integrat de llengües que té l'eusquera d'eix. L'objectiu d'aquest treball consisteix a analitzar com s'insereix en aquest context la didàctica de la gramàtica. Després de lanàlisi dels materials de Llengua Castellana, Eusquera $i$ Anglès, es conclou que els principis metodologics són adequats, tot $i$ que pel que fa a lensenyament de la gramàtica, es troba a faltar més desenvolupament de la reflexió metalingüistica $i$ més aprofitament de la comparació entre llengües.

Paraules clau: didàctica de la gramàtica; tractament integrat de llengües; multilingüisme; plurilingüisme; reflexió metalingüistica. 


\section{Introducción}

En el año 2002 se adopta en la Unión Europea el programa de trabajo "Educación y formación 2010", que da lugar al marco estratégico para la cooperación europea en el ámbito de la educación y la formación (Education and Training [ET] 2020). Teniendo en cuenta ese marco, así como los retos educativos propios de la Comunidad Autónoma del País Vasco (CAPV), el Gobierno Vasco pone en marcha el Plan «Heziberri 2020», cuyo objetivo esencial consiste en mejorar la calidad del sistema educativo de esta comunidad autónoma. El plan consta de tres proyectos: 1) la elaboración del modelo educativo pedagógico; 2) la elaboración de los decretos curriculares; y 3) la elaboración y aprobación de una Ley Vasca de Educación (aún en proceso). En el ámbito de la Educación Primaria, en 2016 sale a la luz el nuevo decreto curricular para la enseñanza básica (Decreto 236/2015). Este nuevo decreto prioriza la adquisición por parte del alumnado de las competencias básicas, que pueden ser transversales o disciplinares. Entre las cinco competencias básicas transversales se menciona en primer lugar la competencia para la comunicación verbal, no verbal y digital, y entre las siete competencias básicas disciplinares la primera de todas es la competencia en comunicación lingüística y literaria, lo que da una idea de la relevancia que este currículum concede a la formación lingüística.

$\mathrm{Al}$ amparo de este nuevo marco educativo, diferentes editoriales han impulsado la adaptación o creación de nuevos manuales. Entre las diferentes iniciativas destaca el Proyecto EKI ${ }^{1}$, elaborado por la editorial IkasElkar, nueva empresa editora que resulta de la fusión de la editorial Elkar y la Federación de Ikastolas². Siguiendo los principios metodológicos del plan Heziberri 2020 y del currículum, el Proyecto EKI se basa en las competencias, plantea la enseñanza a partir de situaciones reales de uso, gira en torno al euskara y propone un tratamiento integrado de las lenguas.

El objetivo de este artículo es analizar el tratamiento de la gramática en estos nuevos materiales creados dentro del Proyecto EKI, que se encuentra en proceso de implantación. En concreto, se analizan los manuales de sexto curso de Educación Primaria referidos a la enseñanza de las diferentes lenguas (Lengua Castellana, Euskara e Inglés), unos manuales que se utilizaron por primera vez durante 2017-2018 (los materiales de quinto curso se introdujeron en el 2018-2019 y en el 2019-2020 se han implantado los correspondientes al cuarto curso).

El análisis viene motivado por el papel fundamental que en las últimas décadas otorgan algunos estudios al conocimiento explícito de la gramática en el aprendizaje de lenguas (Camps, Guasch, Milian y Ribas, 2005; Camps y Milian, 2017; Ellis, 1994; Ribas, Fontich y Guasch, 2014; Wright y Bolitho, 1993; entre otros), y que aparece reflejado de manera más o menos explícita en el propio currículum para la enseñanza básica de la CAPV.

El análisis se centra específicamente en el lugar que se da a la didáctica de la gramática, prestando atención a tres aspectos que adquieren especial relevancia en la metodología de EKI,

1 EKI son las siglas de Euskal Curriculuma, Konpetentzietan oinarrituta, Integrazioaren pedagogiaz [Curriculum Vasco, basado en Competencias, sobre la pedagogía de la Integración]. Asimismo, la palabra eki significa "sol" en euskara.

2 El material del proyecto EKI ha sido diseñado para ser utilizado también en regiones como Navarra. El análisis que aquí presentamos, no obstante, se circunscribe al contexto de la CAPV y al marco educativo Heziberri 2020 de reciente implantación en esta comunidad. 
en respuesta a las orientaciones recogidas en el marco pedagógico educativo Heziberri 2020 para el desarrollo del plurilingüismo (Gobierno Vasco, 2014:34-35) y al propio decreto curricular elaborado dentro de dicho marco: 1) el enfoque basado en los géneros textuales, que plantea una enseñanza de la lengua basada en situaciones de uso $^{3} ; 2$ ) el tratamiento integrado de lenguas, aspecto esencial en una comunidad autónoma con dos lenguas oficiales no vinculadas genéticamente; y 3 ) la reflexión metalingüística, que constituye uno de los seis bloques de contenido que el currículum vasco define para la enseñanza de la lengua.

\section{La metodología del Proyecto EKI y de Heziberri 2020 en torno a la enseñanza de la lengua y los contenidos gramaticales}

Siguiendo los fundamentos de Heziberri 2020, el material didáctico EKI está específicamente orientado a la educación basada en competencias. «El alumnado debe conseguir la competencia en cualquiera de las situaciones cotidianas de la vida: en el pensamiento y en el estudio, en la comunicación, en la convivencia, en el desarrollo de su propia personalidad, así como en la acción y el emprendizaje» (IkasElkar, s.f.a). Es por ello que la enseñanza de la lengua en general, y la de los contenidos gramaticales en particular, está vinculada a situaciones comunicativas reales, de manera que la transmisión de los contenidos lingüísticos o gramaticales no es un fin en sí mismo, sino un medio para resolver dichas situaciones comunicativas.

A continuación, se explican los tres aspectos metodológicos en los que se centrará nuestro análisis: el enfoque basado en los géneros, el tratamiento integrado de lenguas y la reflexión metalingüística.

\subsection{Enfoque basado en los géneros textuales}

El enfoque metodológico de EKI, según afirman sus autores, está basado en el enfoque sociodiscursivo de Bronckart (1997), fundamentado en la pedagogía textual. Los textos se definen como unidades de comunicación que toman forma y sentido en el contexto de una situación comunicativa concreta y los géneros textuales, a su vez, se definen como las formas textuales identificadas por los hablantes de una comunidad. Entre las ventajas del enfoque basado en géneros, los autores destacan la posibilidad de integrar con flexibilidad y fraccionar los elementos del texto: textos completos, secuencias discursivas para desarrollar fragmentos de un texto, los componentes del texto que permiten desarrollar sus secuencias discursivas (narrativas, descriptivas, explicativas, argumentativas...) y, finalmente, los elementos lingüísticos empleados para formar estas secuencias (IkasElkar, s.f.b.).

Este enfoque concuerda plenamente con la visión de la lengua perceptible en el currículum creado a partir del Plan Heziberri 2020, donde la didáctica de la lengua se entiende dentro de un

3 El marco pedagógico de Heziberri 2020 no propone exactamente un enfoque basado en géneros textuales, aunque sí pone de manifiesto la necesidad de «abordar una metodología que asuma el proceso de aprendizaje guiado por proyectos de comunicación significativos, que haga del texto la unidad comunicativa fundamental» (Gobierno Vasco, 2014:35). 
enfoque comunicativo, en consonancia con el cual la unidad lingüística fundamental es el texto en lugar de la oración o el párrafo, una idea proporcionada desde la Lingüística del Texto.

Desde el punto de vista de la didáctica de la gramática, el enfoque basado en los géneros o tipos de discurso resulta positivo, fundamentalmente porque

amplía los ámbitos del aprendizaje gramatical, tradicionalmente limitado a unidades como la oración y la palabra, y nos aporta criterios para la selección de contenidos gramaticales relevantes en los dominios de la coherencia y de la cohesión del texto. (Zayas Hernando y Rodríguez Gonzalo, 1992:s.p.)

\subsection{El tratamiento integrado de lenguas}

Uno de los principios metodológicos de EKI es desarrollar un tratamiento «integral e integrador de las lenguas, garantizando el conocimiento del euskara y su uso adecuado exigido para cada situación» (IkasElkar, s.f.a.). El objetivo es que el alumnado sea capaz de comunicarse eficazmente (aunque en distinto grado) en las lenguas oficiales de la comunidad y en, al menos, una lengua extranjera.

La educación plurilingüe implica que las enseñanzas de las distintas lenguas compartan el objetivo común de desarrollar la competencia comunicativa y que los aprendices utilicen las lenguas para adquirir nuevos conocimientos (Guasch, 2008; Ruiz Bikandi, 2008). Se asume, además, que la enseñanza de lenguas de forma integrada implica una interdependencia entre las lenguas que deriva habitualmente en una transferencia positiva de destrezas y conocimientos, aunque también de carencias; no obstante, la subsanación de las carencias es posible por medio de la propia transferencia, es decir, «desde el aprendizaje en una lengua» (Larringan, Idiazabal y García-Azkoaga, 2015:169). Esto último implica que impulsar la transferencia positiva de conocimientos entre las lenguas es una forma apropiada de plantear la enseñanza de las lenguas (Larringan et al., 2015).

La relevancia de la transferencia se explica a través de la hipótesis de la interdependencia lingüística formulada por Cummins (1981), que predice la transferencia bidireccional de destrezas entre la L1 y la(s) L2. Las condiciones que Cummins (1981) establece para que se dé esta transferencia son la adecuada exposición a la lengua y la motivación del aprendiz por aprender las lenguas. Y es precisamente esta actitud favorable hacia la diversidad lingüística uno de los aspectos que tratan de promover el tratamiento integrado de lenguas (TIL) y, en general, las nuevas metodologías de la educación plurilingüe, que contemplan la interacción de los diferentes sistemas lingüísticos presentes en la mente del aprendiz multilingüe como estrategia para fomentar la transferencia entre lenguas (Cenoz, 2017; Cummins, 2017; García, 2011; entre otros).

De hecho, según el currículum de Educación Primaria de la CAPV, el TIL plantea una enseñanza de las lenguas basada en la inclusión, en el uso, en el enfoque comunicativo y «en el desarrollo de actitudes positivas hacia las lenguas y los hablantes» (Decreto 236/2015, art. 11.2). Esto es coherente con los objetivos y los contenidos que se definen dentro de las áreas dedicadas a la enseñanza de la lengua. Así, entre los objetivos de Lengua Vasca y Literatura y de Lengua Castellana y Literatura se incluye «[r]econocer la diversidad lingüística del centro y del entorno 
para adoptar una actitud positiva hacia ella» y entre los contenidos se definen seis bloques, el sexto de los cuales se centra en la dimensión social de la lengua (Decreto 236/2015:161-162).

En concreto, en el decreto curricular para la Educación Básica de la CAPV, el TIL se entiende como «una estrategia metodológica que presta especial atención a la transferencia de los aprendizajes en distintas lenguas» y que consiste en "trabajar en cada una de las lenguas lo que le es propio y compartir entre todas lo que tienen en común» (Decreto 236/2015:art. 11.1). En consecuencia, el currículum crea, partiendo de un enfoque comunicativo, una programación con una metodología común para las tres lenguas, en la que se fijan unos objetivos, bloques de contenido y criterios de evaluación comunes para las lenguas oficiales ${ }^{4}$.

Aunque las lenguas tienen cada una su código independiente, hay muchos elementos susceptibles de una transferencia positiva. En concreto, los ámbitos textuales, discursivos y pragmáticos pueden, por medio de una metodología adecuada, ser transferidos de unas lenguas a otras (Beeman y Urow, 2011; Larringan et al., 2015; Leonet, Cenoz y Gorter, 2017; Roca de Larios y Murphy, 2001) y, en los casos en que la transferencia no se produce, el TIL ofrece la oportunidad de atender a las similitudes y diferencias entre las lenguas, promoviendo así la reflexión interlingüística y favoreciendo una mayor conciencia metalingüística.

\subsection{La reflexión metalingüística}

Si bien en la presentación del material del Proyecto EKI no se hace alusión explícita a la necesidad de fomentar la reflexión sobre la lengua y sus usos, sí se incide en la importancia de adquirir las competencias básicas, tanto disciplinares como transversales, especificadas en el currículum vasco y los objetivos y contenidos relacionadas con estas. Pues bien, al analizar el currículum se ve que uno de los objetivos relacionados con la competencia en comunicación lingüística y literaria es precisamente el de «[r]eflexionar sobre las convenciones y las normas básicas de uso de las lenguas, y aplicarlas en relación con los procedimientos de comprensión y producción textuales para favorecer el uso eficaz de las mismas» (Decreto 236/2015:141). Siguiendo esta línea, el bloque de contenidos 5 se centra en la «[r]eflexión sobre la lengua y sus usos» y, como se explica en el texto del decreto, «integra los contenidos relacionados con la reflexión sobre los elementos que conforman el sistema lingüístico y sobre las normas que rigen su uso» (Decreto 236/2015:141). Como el currículum deja claro, dicha reflexión es esencial para adquirir y desarrollar las habilidades lingüístico-comunicativas. De hecho, entre los criterios de evaluación se incluye, por ejemplo, el de

[r]eflexionar sobre el sistema y las normas de uso de la lengua, mediante la comparación y transformación de textos, enunciados y palabras, y utilizar estos conocimientos para solucionar problemas de comprensión y en los procesos de textualización y revisión dirigida de los textos, (Decreto 236/2015:141)

lo que no deja lugar a dudas acerca de la relevancia de la reflexión metalingüística en el currículum. Que el currículum impulse la reflexión sobre la lengua, su uso y las normas que rigen ese uso no es cuestión baladí, pues con el término gramática se designa el sistema organizativo

4 En el caso de la lengua extranjera, se formulan objetivos, contenidos y criterios de evaluación específicos. 
específico de cada lengua y «[e]l hablante emplea las potencialidades de esta lengua-mecanismo de acuerdo con una conciencia lingüística o metalingüística cuyo nivel varía» (Larringan, 2011:188). Hasta qué punto el conocimiento explícito de la gramática puede ayudar a hacer un mejor uso de la lengua es una cuestión discutida sobre la que ha habido posturas muy variadas. Algunos autores son escépticos ante la idea de que el conocimiento explícito influya positivamente en el aprendizaje de lenguas (Andrews, 2005; Williams, 2005). No obstante, en las últimas décadas se han desarrollado trabajos que apuntan a que, tanto en primeras como en segundas lenguas, el conocimiento de la gramática proporciona un mayor dominio de la lengua y de sus potencialidades (Boivin, Fontich, Funke, García-Folgado y Myhill, 2018; Camps, Guasch, Milian y Ribas, 2005; Camps y Milian, 2017; Ellis, 1994; Fontich, 2019; Rättyä, Awramiuk y Fontich, 2019; Ribas, Fontich y Guasch, 2014; van Rijt, de Swart y Coppen, 2018; Wright y Bolitho, 1993; entre otros).

Parece que se puede afirmar, por tanto, que la reflexión metalingüística influye positivamente en el proceso de aprendizaje de una lengua, tanto en el nivel oral como, muy especialmente, en el nivel escrito. Además, los estudios sugieren que la reflexión metalingüística acelera el aprendizaje de la lengua, aparte de dotar al aprendiz de autonomía en su aprendizaje y de facilitar en él los procesos de transferencia positiva entre las lenguas que conoce, así como la realización de inferencias. Todo esto es precisamente lo que justifica la atención a la forma (Focus on Form) defendida desde distintos enfoques y métodos actuales (Doughty y Williams, 1998; Ellis, 2016; Lyster, 2004).

Por tanto, en razón de los principios metodológicos arriba expuestos, lo que cabe esperar del material que aquí se analiza es: 1) que los contenidos gramaticales se seleccionen atendiendo a las necesidades lingüísticas derivadas de una competencia global relacionada con una situación comunicativa determinada (la comprensión y creación de un tipo de texto concreto); 2) que, tal y como se define en el TIL, se atienda a las similitudes y diferencias existentes entre las diferentes lenguas y, por lo tanto, se fomente también la reflexión interlingüística; y 3) que a la hora de trabajar los contenidos gramaticales se fomente la conciencia metalingüística.

\section{Análisis de los contenidos gramaticales en el Proyecto EKI}

El material de EKI está formado por los libros del alumno, los correspondientes libros del profesor y materiales complementarios. Para la elaboración de este artículo se han analizado únicamente los libros del alumno (Aiesta, 2017; Aldasoro y Barrutia Arregi [en lo sucesivo ABA], 2017; Lindsay, 2017). Cada manual consta de tres cuadernos (uno por cada trimestre), cada uno relacionado con una competencia global. En el caso de las lenguas, esta competencia gira en torno a una tipología textual.

Para realizar el análisis del material y proporcionarle una base teórica (Tomlinson 2010:87-97), se ha establecido una lista de criterios en función de los principios metodológicos expuestos en el apartado anterior y se han recogido datos cualitativos de todas las actividades en las que se trabajan contenidos gramaticales en cada una de las lenguas, realizando una posterior comparación y extrayendo las principales fortalezas y debilidades. 


\subsection{Enfoque basado en los géneros textuales}

Como se ha explicado, el proyecto EKI recurre al enfoque basado en los géneros ${ }^{5}$, que observa las regularidades que existen en los textos en función de su género y utiliza ese conocimiento para enseñar la lengua escrita. Este enfoque ofrece la ventaja de que la lengua se contempla en su dimensión comunicativa, por lo que los fenómenos gramaticales adquieren sentido al servir a la finalidad del tipo de texto en el que aparecen (Alexopoulou, 2010:102). Esto no implica dejar de prestar atención a la gramática; es más, la didáctica de la gramática ocupa un lugar específico dentro de este enfoque, ya que tanto la estructura del texto en sí, como la estructura interna de las oraciones que lo componen, son esenciales para la coherencia del texto.

La aplicación del enfoque textual, por lo tanto, debe hacerse sin descuidar ninguno de los componentes del texto (extralingüísticos y lingüísticos). Y es precisamente en relación a esta cuestión donde se han detectado posibles áreas de mejora.

En primer lugar, teniendo en cuenta que uno de los objetivos de este enfoque es precisamente que el alumnado aprenda a componer y escribir textos en función de su tipología, sería conveniente que se trabajara la identificación de la tipología del texto (Alexopoulou, 2010:105); sin embargo, la clasificación de tipos y géneros discursivos no siempre es del todo clara en los materiales analizados. Si bien en el primer trimestre de lengua castellana se trabaja específicamente sobre el relato o cuento y sus características tanto a nivel discursivo como estructural, en el segundo y tercer trimestre la tipología textual es en ocasiones confusa. Por ejemplo, en el segundo trimestre (ABA, 2017) se comienza trabajando una serie de historias o mensajes autobiográficos cuya tipología no está clara (pp. 16-22), y se termina con noticias y reportajes periodísticos (pp. 57-63). En el tercer trimestre, por su parte, parecen tratarse los discursos informativos, pero se habla de «informar para imaginar» (ABA, 2017:12), se introduce entre los textos utilizados una carta (p. 16), así como textos descriptivos (p. 19), porque «[1] a actividad de informar se realiza frecuentemente por medio de descripciones» (p. 24) y, finalmente, se dice que «[1] a actividad de informar consiste a menudo en dar pautas o instrucciones sobre cómo realizar algo» (p. 44), lo que se toma como punto de partida para pasar al texto instructivo.

Aparte de una mayor claridad en la diferenciación e identificación de los tipos y géneros discursivos trabajados, sería asimismo deseable una mayor atención a la dimensión estructural de la lengua, y fundamentalmente una mayor atención a la gramática, ya que todo texto tiene una dimensión comunicativa, una dimensión pragmática y una dimensión estructural (que depende de la consideración de reglas gramaticales) (Alexopoulou, 2010:99).

Tal y como cabe esperar, en el material de EKI los contenidos gramaticales seleccionados muestran coherencia con la tipología textual sobre la que se trabaja. Por ejemplo, al trabajar el cuento, se presta atención a las formas gramaticales de primera y tercera persona (pronombres personales, posesivos, terminaciones verbales...) a fin de desarrollar una mayor conciencia sobre

\footnotetext{
5 En el material analizado, se utilizan indistintamente tanto tipos como géneros de discursos. Sin embargo, existe una diferencia entre tipo y género discursivo. Los tipos (descriptivo, narrativo, expositivo, argumentativo e instructivo) son estables, aunque no se ha llegado a un acuerdo sobre su clasificación. Los géneros, por el contrario, son variables y se van configurando en función de los cambios sociales, lo que explica el surgimiento de nuevos géneros, como el chat, el email, etc. (Alexopoulou, 2010:100).
} 
los tipos de narrador que existen, al uso del guion largo o raya en los diálogos, etc. Sin embargo, en ocasiones no se profundiza lo suficiente en cuanto a cómo y cuándo usar los elementos lingüísticos frecuentes en un tipo de texto determinado, los cuales se presentan como opciones equivalentes obviando sus diferencias sintácticas y semánticas. Por ejemplo, al trabajar el texto argumentativo (Euskara, tercer trimestre: Aiesta, 2017:23), el manual propone distintas opciones para expresar la causa: oraciones subordinadas causales (bait-, -(e)lako, eta), conectores causales (horregatik, 'por eso') e incluso elementos que sin ser exactamente causales pueden servir para aducir una razón, como la locución adverbial izan ere 'de hecho' o los dos puntos. Efectivamente, desde un punto de vista pragmático, todas estas opciones son válidas. No obstante, debe tenerse en cuenta que no son sintáctica y semánticamente equivalentes, y que, al no prestar atención a sus diferencias sintácticas y semánticas, se corre el riesgo de conceptualizar y clasificar estos elementos de manera incorrecta.

Además, si bien se tiene presente la adecuación del discurso al contexto comunicativo, otras cuestiones relevantes, como el registro, no siempre se tienen en consideración. Por ejemplo, en relación con el texto instructivo se presentan cuatro opciones, a saber, uso del imperativo, del infinitivo, del verbo en presente y primera persona del plural, y de construcciones con SE (no se habla de construcciones impersonales) (Lengua Castellana, tercer trimestre: ABA, 2017:48). Puesto que todas estas opciones permiten transmitir instrucciones, se presentan como equivalentes, sin diferenciar, por ejemplo, entre registros formales e informales.

Un nuevo ejemplo se encuentra en los textos destinados a 'dar razones' (Euskara, tercer trimestre: Aiesta, 2017:39-40). En esa unidad se explica que el autor de un texto se puede mostrar a sí mismo o puede esconderse (se refiere a utilizar un estilo personal o impersonal). A partir de ese momento se le plantean al alumno situaciones varias y se le pide que dé razones en ellas mostrándose u ocultándose:

Jende askok esaten du aspertuta dagoela beti ni, ni, gu, gu irakurtzeaz; batzuek beren burua ezkutatuz bidali nahiko lukete testu hori. Hortaz, berridatzi egingo duzu testua, baina, oraingoan, zeure burua ezkutatuz. [Mucha gente dice que está aburrida de leer siempre yo, yo, nosotros, nosotros; algunos preferirían enviar este texto escondiéndose a sí mismos. Por tanto, escribe de nuevo el texto, pero ahora escondiéndote] (Euskara, tercer trimestre: Aiesta, 2017:40).

Es decir, la razón que se da para elegir el estilo impersonal es la de evitar el hartazgo, obviando diferencias significativas derivadas del registro o contexto. A través de ejemplos y explicaciones sencillas se podría introducir esta diferenciación, que es esencial dentro del enfoque comunicativo, e introducir terminología específica (i.e., estilo personal e impersonal) que ayude al alumnado a distinguir con mayor claridad los conceptos (véase el punto 2.3).

Resumiendo, el material analizado opta acertadamente por un enfoque basado en tipos y géneros discursivos que permite trabajar los aspectos lingüísticos de manera contextualizada y significativa. Sin embargo, la manera en la que se trabajan los contenidos gramaticales dentro de este enfoque podría mejorarse si se prestara más atención a las diferencias sintácticas, semánticas e incluso pragmáticas de los elementos lingüísticos utilizados, ayudando al alumno a desarrollar una mayor conciencia metalingüística.

En cualquier caso, es justo recordar quela selección e integración de contenidos gramaticales 
en la práctica discursiva es en sí uno de los aspectos de mayor dificultad en la elaboración de materiales. De hecho, no es la primera vez que se pone de manifiesto en el análisis de materiales didácticos el problema de que la enseñanza de la gramática no enlaza con el aprendizaje de los usos discursivos. Zayas (2012:76) se refiere a esta cuestión y recoge la opinión de Anna Camps, según la cual «este es un tema crítico en la enseñanza de las lenguas, donde son más las preguntas planteadas que las soluciones».

\subsection{Tratamiento integrado de lenguas y desarrollo de la conciencia interlingüística}

Como se ha comentado arriba, uno de los objetivos del currículum de Heziberri 2020 es «transferir los conocimientos y estrategias de comunicación adquiridos en otras lenguas y materias» (Decreto 236/2015:12). Además, en consonancia con el currículum, dentro de los principios metodológicos del proyecto EKI se contempla el desarrollo del multilingüismo ${ }^{6}$, con el euskara como eje, y el TIL, trabajando en cada lengua lo que le es propio y compartiendo lo que es común. Dada la posibilidad de transferir conocimientos y destrezas de una lengua a otra (Cummins, 1981; Larringan et al., 2015:168-169), sería esperable encontrar en los materiales cierto paralelismo entre las tres lenguas (castellano, euskara, inglés) en cuanto a los contenidos y al tipo de actividades. Además, cabría predecir la presencia de referencias a las demás lenguas en cada uno de los libros de texto, para fomentar la reflexión interlingüística.

En cambio, hemos observado que, si bien algunos tipos de texto y contenidos son comunes (aunque no todos se trabajan en todas las lenguas ni se hace de manera paralela, lo que hasta cierto punto es comprensible, dada la gran diferencia de nivel entre las dos lenguas oficiales y la extranjera), no se refleja de manera explícita la relación entre las tres lenguas. Es decir, en ningún momento se hace referencia expresa a las similitudes y diferencias existentes en relación a un determinado aspecto lingüístico, a pesar de que determinados rasgos lingüísticos podrían ser, e incluso convendría que fueran, puestos en relación en las tres lenguas; se desaprovecha, por tanto, la oportunidad de integrar las distintas gramáticas.

Por ejemplo, una de las cuestiones centrales en el primer trimestre de Lengua Castellana son los adjetivos y su concordancia. Sin embargo, en las demás lenguas no se trabaja específicamente la gramática del adjetivo, y con ello se pierde una oportunidad valiosa para la comparación interlingüística, ya que las tres lenguas presentan variación en cuanto a la concordancia y al orden de los elementos que conforman el sintagma nominal':

\footnotetext{
6 Multilingüismo y plurilingüismo son dos conceptos que el MCER (2002:4-5) diferencia nítidamente. El proyecto EKI tiene como objetivo el multilingüismo. Sin embargo, el concepto de plurilingüismo implica un punto de vista más amplio y ambicioso, y en nuestra opinión más en consonancia con los verdaderos objetivos del sistema educativo.

7 Det. $=$ determinante masc. $=$ masculino; pl. $=$ plural; sust. $=$ sustantivo adj. $=$ adjetivo.
} 


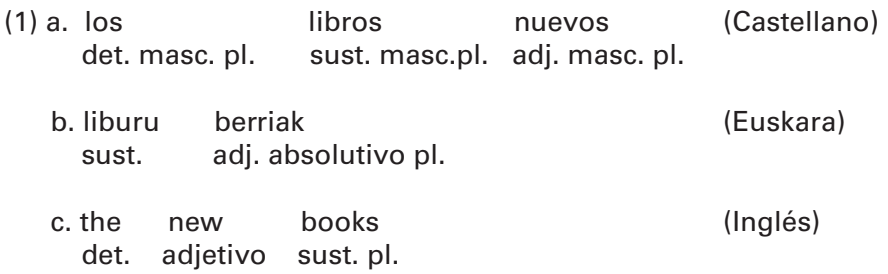

Como se observa en (1), mientras que en castellano todos los elementos concuerdan tanto en número cómo en género (1a), en inglés solamente existe marcado de número y sólo se manifiesta en el elemento final del sintagma (1c); lo mismo sucede en euskara (1b): el elemento final lleva marca de número, y al contrario que en las otras dos lenguas, lleva también marca de caso (berri 'nuevo/a' + -ak 'absolutivo plural'). Dado que estas diferencias en la gramática de las tres lenguas son un posible foco de error o transferencia negativa (Larsen-Freeman y Long, 1991), sería conveniente poner especial atención en ellas y fomentar así un mayor grado de conciencia interlingüística.

Otro claro ejemplo en el que podría trabajarse la reflexión interlingüística es el de las oraciones interrogativas. En EKI se trabajan en castellano e inglés independientemente (en euskara no se llegan a trabajar siquiera); sin embargo, no se presta atención a sus similitudes (i.e. la morfofonología de las palabras interrogativas, la inversión sujeto/verbo), tal y como se muestra en (2), ni a sus diferencias (i.e. presencia/ausencia de verbo auxiliar), como se advierte en (3) ${ }^{8}$.

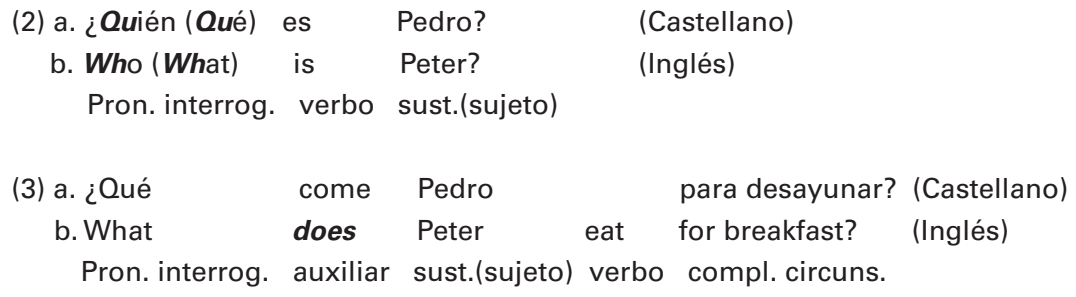

Así, a pesar de las ventajas que la especial situación sociolingüística de la CAPV aporta al TIL, se desaprovecha la oportunidad de reflexionar sobre las relaciones entre las gramáticas de esas tres lenguas (genéticamente no emparentadas) y de identificar lo que tienen en común y lo que no (lo que facilitaría la realización de transferencias positivas y contribuiría a evitar posibles transferencias negativas). Es decir, se pierde una ocasión propicia para desarrollar una mayor conciencia interlingüística, que redunde en un mayor dominio de la lengua y distinción de códigos (Ruiz Bikandi, 2005, 2008).

\subsection{La reflexión metalingüística}

Con el auge del enfoque comunicativo, la enseñanza de la gramática se ha ido relegando ante la creencia de que trabajar los contenidos gramaticales no es efectivo (Andrews, 2005) y que es mediante su uso como realmente se aprende la lengua. Hoy en día esta postura está superada

8 Pron. interrog. $=$ pronombre interrogativo; sust. $=$ sustantivo; compl circuns. $=$ complemento circunstancial. 
y diferentes autores convienen en que los contenidos gramaticales y, en particular, la reflexión sobre la forma y el funcionamiento de la lengua es necesaria y debe integrarse dentro del enfoque comunicativo (Camps et al., 2005; Camps y Milian, 2017; Ellis, 1994; Fontich y García-Folgado, 2018; Ribas et al., 2014; Wright y Bolitho, 1993; entre otros). El mismo decreto curricular para la Educación Básica de la CAPV (Decreto 236/2015) incide, como se ha mostrado, en la necesidad de esta reflexión, proponiendo un bloque entero de contenidos en torno a ella.

En este apartado analizamos las actividades en las que se trabaja la gramática, prestando atención a dos aspectos: 1) si éstas entrañan procesos de metacognición y, concretamente, de reflexión metalingüística, y 2) si la terminología empleada y las definiciones y explicaciones ofrecidas facilitan el razonamiento metalingüístico.

\subsubsection{Procesos de metacognición y reflexión}

En los materiales analizados se aprecia un esfuerzo por fomentar la reflexión a través de diferentes tipos de actividades que buscan desarrollar la metacognición (actividades de investigación, autorregulación...). La mayoría de estas actividades se integran en el proceso de escritura de un texto y se centran, sobre todo, en aspectos discursivos y textuales. Por ejemplo, en la unidad 6.3 de Lengua Castellana se pide a los alumnos que hagan hipótesis sobre quiénes podrían ser el emisor y el destinatario de un determinado texto (Lengua Castellana, tercer trimestre: ABA, 2017:15-21), cuál es la intención comunicativa del texto (ibid.:22), cómo se organiza la información a través de párrafos (ibid.:30-36), etc., y finalmente los alumnos deben autorregular su aprendizaje haciendo una revisión del texto mediante una rúbrica (ibid.:37). Un menor número de actividades están enfocadas a trabajar aspectos ortográficos, morfológicos o sintácticos. En la mayoría de ellas no se desarrolla la reflexión, y en las muy escasas ocasiones en las que se hace, se aprecian ciertas carencias metodológicas.

Véase primero un ejemplo en el que se anima al alumno a la reflexión, a fin de inferir la regla de acentuación de las palabras interrogativas (Lengua Castellana, primer trimestre: ABA, 2017:23):

Copia todas las preguntas que se te han formulado en la actividad 5 sobre el mito El cuervo roba la luz. [...] Observa todas las preguntas; teniendo en cuenta las palabras de inicio que llevan tilde, ¿cuál de las siguientes conclusiones te parece más acertada?

a) La primera palabra de una pregunta lleva tilde.

b) Palabras como que, quien, como, cuando o donde siempre llevan tilde.

c) Palabras como que, quien, como, cuando o donde llevan tilde cuando son el elemento clave de una pregunta.

A primera vista la actividad entraña diferentes procesos metacognitivos por parte del alumno: observar ciertos datos y hacer hipótesis acerca de cuál es la conclusión acertada. Sin embargo, en la lista de preguntas que el alumno debe analizar para extraer la conclusión correcta no hallamos ejemplos contrastivos en los que palabras como que, quien, como, cuando o donde no deban ser acentuadas, ni ejemplos en los que que, quien, como, cuando... formen parte de una oración interrogativa indirecta. Es decir, no se ofrece un conjunto de datos significativos que 
faciliten que el alumno tome conciencia del contraste entre los diferentes usos de estas palabras y su relación con la acentuación.

Asimismo, se plantean numerosas actividades en las que el alumno no tiene necesidad de recurrir a procesos de reflexión para llevar a cabo el objetivo de la tarea. Sin ir más lejos, en las actividades que suceden a la actividad del ejemplo anterior, los alumnos tienen que rellenar huecos con las palabras que, como, cuando... o escribir preguntas adecuadas para una serie de respuestas. El objetivo es que el alumno acentúe correctamente las palabras interrogativas, aplicando la regla que se ha inferido previamente. A pesar de que en el enunciado de la actividad se dice explícitamente, en alusión a la tilde, que estas palabras «unas veces la llevan y otras no» (Lengua Castellana, primer trimestre: ABA, 2017:46), el alumno no necesita reflexionar para discriminar entre los casos en los que llevan tilde y los que no, ya que todas las frases comprenden oraciones interrogativas directas.

Otro ejemplo a destacar es el de las actividades dirigidas a expresar hipótesis o contrafactualidad a través de las formas condicionales del verbo. En una primera actividad (de investigación), los alumnos deben relacionar la prótasis de diferentes oraciones condicionales con su correspondiente apódosis «de manera que el resultado sea comprensible» (Lengua Castellana, tercer trimestre: ABA, 2017:25). Aunque las oraciones se agrupan y resaltan en diferentes colores teniendo en cuenta el tipo de condicional al que corresponden (simple o compuesta), no se presta atención a las diferencias semánticas entre los diferentes tipos de oraciones, ni a su correlación con las formas verbales (tiempo y aspecto) empleadas.

A esta actividad le suceden otras dos en las que el alumno debe completar la conjugación de los verbos condicionales acordándose de poner la tilde y rellenar los huecos de las oraciones conjugando el verbo correctamente (Lengua Castellana, tercer trimestre: ABA, 2017:26). Se desaprovecha así la ocasión de reflexionar sobre las diferentes formas de condicional (simple y compuesto) y sus significados.

En otra ocasión, se presentan de manera deductiva la definición de adjetivo y la regla de concordancia. Posteriormente, el alumno debe rellenar huecos seleccionando el adjetivo adecuado, atendiendo a su concordancia con el sustantivo al que modifica, en una actividad que podría calificarse de excesivamente fácil para alumnos nativos de sexto de primaria (Lengua Castellana, primer trimestre: ABA, 2017:58):

Selecciona el adjetivo adecuado para completar cada una de las siguientes frases:

1. Me parecía que una sombra ....... me envolvía.

negro negra negros negras

2. Me han regalado unos zapatos y unas botas ........

rojo roja rojos rojas

En lugar de esta actividad, podría realizarse un análisis contrastivo de la concordancia en las tres lenguas, favoreciendo una mayor conciencia meta- e interlingüística de este fenómeno.

\subsubsection{Adecuación explicativa y uso de la terminología}

Si bien diferentes autores admiten que los problemas terminológicos no son los más importantes en una disciplina (Bosque, 1989:11-12) y que «lo verdaderamente importante no es poner etiquetas 
a los problemas que se estudian sino comprenderlos» (Gutiérrez Rodríguez, 2018:215), existe un amplio consenso en cuanto a la necesidad de una mayor precisión, homogeneidad y unificación de la terminología a fin de evitar problemas de tipo conceptual. Como apunta Camps (2014), un modelo educacional basado en la reflexión y, en concreto, en la reflexión metalingüística, debe tener en cuenta que los alumnos deben gradualmente desarrollar la capacidad de reflexionar sobre la lengua usando un metalenguaje específico que les ayude a consolidar las nociones lingüísticas. La relación de interdependencia entre metalenguaje o terminología y la compresión de las nociones a las que hace referencia es también descrita por Eguren y Fernández Soriano (2006:9), quienes afirman que «la terminología de una disciplina es, entre otras cosas, un reflejo formal de su organización conceptual» y que «[c]uando aprendemos los términos de una materia estamos aprendiendo, por tanto, los contenidos de dicha materia». Estos mismos autores también señalan la necesidad de que las definiciones empleadas sean «precisas» y nos permitan distinguir con claridad unos conceptos de otros.

En esta misma línea, el currículum de Educación Básica de la CAPV recoge entre los criterios de evaluación el de «[c]onocer la terminología lingüística trabajada en el ciclo, y utilizarla en las actividades de reflexión sobre el uso de la lengua asociadas a tareas de comprensión y producción» (Decreto 236/2015:163).

Sin embargo, en el material de EKI para sexto de primaria, se observa que a menudo se evita el uso de terminología específica o se recurre a términos poco precisos y a definiciones que carecen de adecuación explicativa.

Un claro ejemplo de ausencia de terminología precisa es el de las actividades en las que se trabajan las oraciones condicionales, en las que en ningún momento se usa el término «condicional». En su lugar, se usan expresiones alternativas como «estructuras que nos ofrece la lengua para construir de manera correcta eso que imaginamos, que sabemos que no sucedió, pero que si hubiésemos podido...» o «expresiones Si yo...» (Lengua Castellana, tercer trimestre: ABA, 2017:30).

Los problemas a la hora de definir y explicar conceptos gramaticales son especialmente patentes en el caso de las categorías gramaticales, ya sea por su ausencia, por su imprecisión o por la falta de criterios unificados.

Por un lado, categorías como determinante, pronombre o adverbio no aparecen definidas; si bien se trabajan desde varios puntos de vista: como elementos de cohesión dentro de un texto (Lengua Castellana, tercer trimestre: ABA, 2017:38-42), para identificar al narrador (Lengua Castellana, primer trimestre: ABA, 2017:38; Euskara, tercer trimestre: Aiesta, 2017:39), para diferenciar el estilo personal y el impersonal (Euskara, segundo trimestre: Aiesta, 2017:39-40) etc. En ocasiones se alude a ellas con el término genérico "palabras". Otras veces se usan términos específicos como "pronombre», «posesivo» (Lengua Castellana, primer trimestre: ABA, 2017:38; Lengua Castellana, segundo trimestre: ABA, 2017:51), pero sin llegar a definirlos. No es este el caso de las categorías gramaticales adjetivo, verbo y sustantivo, que sí aparecen definidas, aunque no de manera unificada.

La primera vez que se trabajan los adjetivos, verbos y sustantivos es en la unidad del primer 
trimestre de Lengua Castellana (ABA, 2017:52), en una actividad que tiene como objetivo clasificar las palabras en tres cajas (la caja de los adjetivos, la de los verbos y la de los sustantivos). En esta primera ocasión, los alumnos no disponen de ninguna definición de estas categorías gramaticales, y no es hasta más tarde, en la misma unidad (ibid.:58), cuando se ofrece la primera definición de una categoría gramatical: la de los adjetivos. Estos se definen como «palabras que nos sirven para indicar las cualidades, rasgos o propiedades de los nombres a los que acompañan [y] concuerdan en género y número con el nombre al que acompañan, tanto si van delante como si van detrás». El hecho de que en la definición se empleen tanto criterios nocionales como formales supone un acierto, ya que ello permite delimitar y reconocer las distintas categorías con mayor claridad (Eguren y Fernández Soriano, 2006); no obstante, sería conveniente que hubiera menor distancia o mayor relación con la actividad de clasificación anterior (podría, por ejemplo, hacerse referencia a las posibles dificultades surgidas en el ejercicio de clasificación y analizar las palabras de la caja de los adjetivos en base a los criterios que los definen). Es más, en el segundo trimestre (p. 33) se propone nuevamente una actividad de clasificación de palabras en nombres, adjetivos y verbos, pero no solo no existe referencia explícita a la definición de adjetivo sino que las categorías nombre y verbo continúan sin ser definidas. Finalmente, es en los manuales del segundo y tercer trimestre de inglés (Lindsay, 2017) en los que las categorías verbo y sustantivo se encuentran definidas, esta vez usando criterios puramente nocionales. Es decir, los verbos se definen como «action words», los sustantivos como «naming words» y los adjetivos como «describing words». Este tipo de definición nocional es insuficiente para delimitar categorías y, de hecho, como señalan Eguren y Soriano (2006), tiene el inconveniente de que «existen numerosísimas excepciones: nombres del tipo de invasión o atropello denotan acciones y otros como bondad o blancura, cualidades, verbos como saber o tener no son de acción, adjetivos como mero o los llamados «adjetivos determinativos», como mismo, no denotan cualidades, y así un largo etcétera».

En resumen, a la hora de definir las principales categorías gramaticales sería deseable que en las diferentes lenguas se emplearan criterios adecuados y unificados, y que existiera una relación entre actividades que permitiera trabajar la reflexión metalingüística en torno a estas categorías.

\section{Conclusión}

Estructurar la enseñanza de la lengua en torno a tipos y géneros discursivos que están vinculados con situaciones comunicativas constituye un punto de partida adecuado, y en los materiales analizados se observan grandes aciertos, como el de proponer una selección y secuenciación de actividades dirigidas a desarrollar paulatinamente diversas estrategias metacognitivas y metalingüísticas, como la planificación, estructuración, revisión, autocorrección, autoevaluación... en todos los niveles (lingüístico, textual y discursivo) (Camps, 1994; 2014). Todo ello, además, a través del trabajo grupal, lo que promueve la verbalización y el diálogo sobre el proceso de escritura y la propia escritura colaborativa (Camps, 2014:29). Sin embargo, en lo que respecta a la selección e integración de contenidos gramaticales en el enfoque basado en géneros, hemos detectado 
que no se presta suficiente atención a las diferencias sintácticas, semánticas o pragmáticas de los elementos lingüísticos susceptibles de aparecer en un mismo tipo de texto, $y$, por tanto, no se favorece el desarrollo de la conciencia metalingüística.

En aras de una mejor aplicación del enfoque de géneros, consideramos que sería conveniente prestar mayor atención a tres aspectos esenciales: 1) crear una clasificación de discursos más clara y definida; 2) establecer una relación explícita entre los tipos de textos trabajados en las tres lenguas o, en su defecto, interrelacionar aquellos aspectos comunes que permitan transferir los conocimientos y estrategias empleadas para la comprensión y escritura de determinado tipo de textos de una lengua a otra; y, sobre todo, 3) prestar más atención, como recomienda Alexopoulou (2010), a cada uno de los tres planos de organización del texto: su estructura formal, el contenido semántico y los componentes de la microestructura, entre los que se incluye la gramática.

Por su parte, el TIL constituye un punto de partida metodológico esencial en una comunidad autónoma en la que el sistema educativo incluye dos lenguas oficiales y, al menos, una lengua extranjera, todas ellas genéticamente dispares. Sin embargo, no hemos podido encontrar, al menos en el ámbito de la gramática, ninguna alusión explícita en un manual a algo trabajado en otra lengua, aunque, sin duda, existen múltiples oportunidades para ello. De hecho, aunque los contenidos en ocasiones coinciden, no se organizan de manera paralela, así que tampoco intuitivamente se favorece la relación entre lenguas. Creemos que se podría: 1) establecer relaciones explícitas entre aspectos gramaticales tales como los presentados en los ejemplos; 2) trabajar algunos contenidos de manera paralela en el tiempo (o incluso trabajarlos en las tres lenguas en un mismo manual); y 3) fomentar la reflexión interlingüística y, con ello, la actitud favorable hacia la diversidad lingüística. Los alumnos podrían llegar a ser conscientes de manera práctica de las ventajas del plurilingüismo y del aprendizaje de segundas (o sucesivas) lenguas, hecho sobradamente probado en la literatura científica (Morales, Calvo y Bialystok, 2013, entre otros).

Por último, a fin de desarrollar una mayor conciencia sobre el uso de la lengua, sería deseable que se incluyeran más actividades que promoviesen la reflexión metalingüística a través de la observación activa, el reconocimiento, el análisis, la contrastación y la manipulación de formas lingüísticas, y menos actividades en las que el alumno solo necesite aplicar deductivamente una regla determinada. Igualmente, sería conveniente que el alumno se fuera familiarizando con el metalenguaje específico que le permita verbalizar, ya sea individualmente o en grupo, aquello sobre lo que ha reflexionado y aquello que ha aprendido, y participar en la denominada habla exploratoria (Fontich 2014; Mercer, 2000). Para ello, creemos que debe evitarse una excesiva simplificación de la terminología y acordar términos unificados que permitan la correcta conceptualización de las diferentes nociones gramaticales (Eguren y Fernández Soriano, 2006; Gutiérrez Rodríguez, 2008).

Pese a todo ello, es de justicia señalar que la instrucción de la gramática es probablemente el área más compleja y polémica dentro de la didáctica de la lengua. Los principios didácticos del Proyecto EKI tienen el atractivo de abordar esa instrucción desde una perspectiva moderna, integrando la gramática en un enfoque discursivo con el fin de lograr un aprendizaje significativo, si bien en la práctica convendría prestar mayor atención a la forma y uso de las diferentes expresiones 
gramaticales que se integran en el discurso y plantear actividades que promuevan explícitamente la reflexión metalingüística e interlingüística.

\section{Agradecimientos}

Investigación financiada por la Universidad del País Vasco (UPV/EHU) a través del grupo de investigación de Lingüística Teórica HITT (GIU18/221) y del Gobierno Vasco a través del grupo consolidado Language and Speech (LASLAB) (IT904-16).

\section{Referencias}

Aiesta, A. (2017). Euskara LH 6. Donostia: Elkar-Ikastolak Zerbitzuak.

Aldasoro, M., y Barrutia Arregi, E. (2017). Lengua Castellana LH 6. Donostia: Elkar-Ikastolak Zerbitzuak.

Alexopoulou, A. (2010). El enfoque basado en los géneros textuales y la evaluación de la competencia discursiva. En Actas del XXI Congreso Internacional de ASELE (pp. 97-110). Salamanca: Universidad de Salamanca.

Andrews, R. (2005). Knowledge about teaching of [sentence] grammar: The state of play. English Teaching: Practice and Critique, 4(3), 69-76.

Beeman, K., y Urow, C. (2011). El Puente: creando conexiones metalingüísticas. Soleado, 4(1), 2-3, 13.

Boivin, M. C., Fontich, X., Funke, R., García-Folgado, M. J., y Myhill, D. (2018). Working on grammar at school in L1 education: Empirical research across linguistic regions. Introduction to the special issue. Special issue Working on grammar at school in L1 education: Empirical research across linguistic regions. L1-Educational Studies in Language and Literature, 18, 1-6.

Bosque, I. (1989). Las categorías gramaticales. Relaciones y diferencias. Madrid: Síntesis.

Bronckart, J. P. (1997). Activité langagière, textes et discours. Pour un interactionisme socio-discursif. París: Delachaux et Niestlé.

Camps, A. (1994). L'ensenyament de la composició escrita. Barcelona: Barcanova.

Camps, A. (2014). Metalinguistic activity in language learning. En T. Ribas, X. Fontich y O. Guasch (Eds.), Grammar at school. Research on metalinguistic activity in language education (pp. 2541). Brusselles: Peter Lang.

Camps, A., Guasch, O., Milian, M., y Ribas, T. (2005). Bases per a lensenyament de la gramática. Barcelona: Graó.

Camps, A., y Milian, M. (2017). Una gramàtica per a l'ensenyament: característiques i objectius. Caplletra. Revista Internacional de Filologia, 63, 217-243.

Cenoz, J. (2017). Translanguaging in school contexts: International perspectives. Journal of Language, Identity \& Education, 16(4), 193-198.

Cummins, J. (1981). The role of primary language development in promoting educational success for language minority students. En California State Department of Education (Ed.), School- 
ing and language minority students: A theoretical framework (pp. 3-49). Los Ángeles: Evaluation, Dissemination and Assessment Center California State University.

Cummins, J. (2017). Teaching for transfer in multilingual school contexts. En O. García, A. M. Y. Lin y S. May (Eds.), Bilingual and multilingual education (pp. 103-115). Suiza: Springer.

Decreto 236/2015, de 22 de diciembre, por el que se establece el currículo de Educación Básica y se implanta en la Comunidad Autónoma del País Vasco. Boletín Oficial del País Vasco, No 9, de 15 enero de 2016. Recuperado de http://www.jusap.ejgv.euskadi.eus/r47-bopvapps/es/ bopv2/datos/2016/01/1600141a.pdf

Doughty, C., y Williams, J. (Eds.) (1998). Focus on form in classroom second language acquisition. Cambridge: Cambridge University Press.

Eguren, L., y Fernández Soriano, O. (2006). La terminología gramatical. Madrid: Gredos.

Ellis, N. (1994). Introduction: Implicit and explicit language learning: an overview. En N. Ellis (Ed.), Implicit and explicit learning of languages (pp. 1-32). San Diego, CA: Academic Press Ellis, R. (2016). Focus on form: A critical review. Language Teaching Research, 20(3), 405-428.

Fontich, X. (2014). Grammar and reflection at school: Checking out the whats and the hows of grammar instruction. En T. Ribas, X. Fontich y O. Guasch (Eds.), Grammar at school. Research on metalinguistic activity in language education (pp. 25-41). Bruselas: Peter Lang.

Fontich, X. (2019). Should we teach first language grammar in compulsory schooling at all? Some reflections from the Spanish perspective. Crossroads-A Journal of English Studies, 24(1), 26-44.

Fontich, X., y García-Folgado, M.J. (2018). Grammar instruction in the Hispanic area: The case of Spain with attention to empirical studies on metalinguistic activity. L1-Educational Studies in Language and Literature, 18, 1-39.

García, O. (2011). Bilingual education in the 21st century: A global perspective. West Sussex: Wiley-Blackwell.

Gobierno Vasco (2014). Heziberri 2020. Marco del modelo educativo pedagógico. Recuperado de http://www.euskadi.eus/contenidos/informacion/heziberri_2020/es_heziberr/adjuntos/Heziberri_2020_c.pdf

Guasch, O. (2008). Reflexión interlingüística y enseñanza integrada de las lenguas. Textos de Didáctica de la Lengua y la Literatura, 47, 20-32.

Gutiérrez Rodríguez, E. (2018). El laberinto de la terminología lingüística en las aulas. ReGroc. Revista de Gramática Orientada a las Competencias, 1(1), 203-235.

IkasElkar (s.f.a.). Proyecto EKI. Recuperado de http://www.ikaselkar.eus/es/eki/eki-informacion/

IkasElkar (s.f.b.). Lengua Castellana y Literatura arloko material berrien ezaugarri nagusiak. Recuperado de https://jakintza.net/wp-content/uploads/LENdiptikoa.pdf

Instituto Cervantes (2002). Marco común europeo de referencia para las lenguas: aprendizaje, enseñanza, evaluación. Madrid: MECD-Anaya.

Larringan, L. M. (2011). Reflexión metalingüística y enseñanza de la gramática. En U. Ruiz Bikandi (Coord.), Didáctica de la lengua castellana y la literatura (pp. 187-204). Barcelona: Graó.

Larringan, L. M., Idiazabal, I., y García Azkoaga, I. M. (2015). Cartas al director escritas en euskera y castellano por jóvenes bilingües. Transferencias y destrezas textuales y discursivas. 
En I. M. Azkoaga e I. Idiazabal (Coords.), Para una ingeniería didáctica de la educación plurilingüe (pp. 147-171). Universidad del País Vasco / Euskal Herriko Unibertsitatea.

Larsen-Freeman, D., y Long, M. (1991). SLA: Types of Data Analysis. En D. Larsen-Freeman y M. Long (Eds.), An introduction to second language acquisition research (pp. 52-80). New York: Longman.

Leonet, O., Cenoz, J., y Gorter, D. (2017). Challenging Minority Language Isolation: Translanguaging in a Trilingual School in the Basque Country. Journal of Language, Identity \& Education, 16(4), 216-227.

Lindsay, D. (2017). English LH 6. Donostia: Elkar-Ikastolak Zerbitzuak.

Lyster, R. (2004). Differential effects of prompts and recasts in form-focused instruction. Studies in second language acquisition, 26(3), 399-432.

Mercer, N. (2000). Words and minds: how we use language to think together. London: Routledge.

Morales, J., Calvo, A., y Bialystok, E. (2013). Working memory development in monolingual and bilingual children. Journal of Developmental Child Psychology, 114(2), 187-202.

Rättyä, K., Awramiuk, E., y Fontich, X. (2019). Introduction to educational linguistics. A special issue on 'What is grammar in L1 education today?' L1 - Educational Studies in Language and Literature, 1-8.

Roca de Larios, J., y Murphy, L. (2001). Some Steps Towards a Socio-cognitive Interpretation of Second Language Composition Processes. International Journal of English Studies, 1(2), 25-45.

Ruiz Bikandi, U. (2005). La reflexió interlingüistica: ajudar a pensar en/amb/sobre tres llengues. Revista de didáctica de la llengua i de la literatura, 38, 51-66.

Ruiz Bikandi, U. (2008). La reflexión metalingüística desde varias lenguas. Textos de didáctica de la lengua y la literatura, 47, 33-45.

Tomlinson, B. (2010). Principles and procedures of materials development. En N. Harwood (ed.) Materials in ELT: Theory and practice (pp. 81-108). Cambridge: Cambridge University Press.

van Rijt, J., de Swart, P., y Coppen, P. A. (2018). Linguistic concepts in L1 grammar education: A systematic literature review. Research Papers in Education, 34(5), 621-648.

Williams, J. N. (2005). Learning without awareness. Studies in Second Language Acquisition, 27, 269-304.

Wright, T., y Bolitho, R. (1993). Language awareness: a missing link in language teacher education? ELT Journal, 47(4), 292-304.

Zayas, F. (2012). Los géneros discursivos y la enseñanza de la composición escrita. Revista Iberoamericana de Educación, 59, 63-85.

Zayas, F., y Rodríguez Gonzalo, C. (1992). Composición escrita y contenidos gramaticales. Aula de Innovación Educativa, 2, 13-16. 